\section{Cell-division sequence motif}

SIR-We wish to point out that a structural motif shared among three transforming protein classes, the papovaviral large-T antigens, the adenoviral ElA proteins and the $\mathrm{v}$ - and $\mathrm{c}$-myc oncoproteins ${ }^{1.2}$ is also present in the E7 transforming proteins of human papillomaviruses ${ }^{3}$ and the CDC25 gene, that is a mitotic regulator of yeast. The region encoding the shared motif has recently been demonstrated to be required by the large - $T$ antigen of simian virus 40 (SV40) both for transforming function and for its specific binding to the 'negative oncoprotein' $\mathrm{RB}^{4}$, the putative human retinoblastoma gene product, to which the E1A protein also binds specifically'. In addition, this region of SV40 large-T, E1A and human c-myc has been shown to be required for cotransformation with ras oncogenes of primary cells (see bibliography in ref. 1).

The motif is:

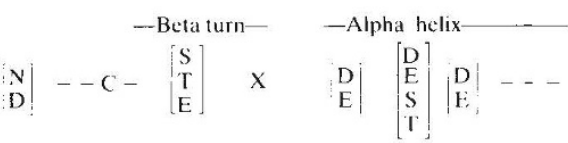

where the brackets indicate alternatives at a given position; the dashes, any amino acid; $\mathrm{N}$, asparagine; $\mathrm{D}$, aspartic acid; $\mathrm{C}$, cysteine; $\mathrm{E}$, glutamic acid; $\mathrm{S}$, serine; $\mathrm{T}$, threonine; and $\mathrm{X}$, a spacer of $1-8$ amino acids, often containing a proline and serines. There is a strong correlation between the presence of an aspartic acid in the first position and a hydrophobic amino acid in the second position. The predicted alpha helix is dominated over the remainder of its length by polar residues. Studies on the SV40 large-T and the adenoviral E1 A proteins show that mutation of the glutamic acid in the sixth position of this descriptor to a positive amino acid inactivates the transforming activity of the proteins'. In one of the $v$-myc oncoproteins, at least one serine/threonine site in this region is phosphorylated". This may also be how the region is activated in normal cellular proteins that contain the motif, while in some oncoproteins activation may be the result of the substitution of serine or threonine by glutamic acid or aspartic acid. This fits the apparent and unusual equivalence of all four amino acids in at least one position in the motif.

Using a sequence regular-expression handler and a modified Chou-Fasman secondary-structure prediction algorithm to annotate sequences with potential secondary structure, all matches to the descriptor within the NBRF protein sequence database (release 16) and the translated Genbank database (release 55) were identified out of over 11,000 proteins. They included nearly all of the $v$-myc and c-myc proteins, nearly all of the ElA and large $T$ proteins, and six of the eight E7 proteins. Additional matches among proteins related to either cell transformation or division include a deer papillomavirus protein, a glycoprotein of Rift Valley fever virus and, perhaps most importantly, the CDC25 protein of yeast?. Only 12 functionally unrelated proteins were clearly identified. It is difficult to assess whether or not the pattern fails to match other known transforming proteins (for example, fos, jun and myb) because their molecular mechanisms are different or because the pattern itself is incomplete. Given the functional complexity of oncogenesis, we currently favour the former and therefore, believe the pattern's specificity to be nearly $100 \%$. In which case, this may be one of the most diagnostic protein functional patterns known.

The importance of the match with the CDC25 protein is that it interacts with a known protein kinase WEE $1+$ and apparently modulates the activity of CDC2, another mitotic regulator protein 7 interestingly, the ability of CDC25 to initiate cell division shows dosage effects, which are compatible with a protein-protein binding titration, such as is now known for the interaction of SV40 large-T or E1A with RB.

Thus, we propose that the majority of proteins containing the motif carry out one of the required steps in the cell division competency cascade of deactivating a cell division repressor (or activating a cell division activator). That these proteins will only transform primary cells in the presence of ras oncogenes suggests that at least one other cell-division signal transduction pathway exists.

JAMES Figge

Dana-Farber Cancer Institute, TEMPLE F. SMITH

Harvard University,

44 Binney Street,

Boston,

Massachusetts 02115, USA

I. Figge, J., Webster. T., Smith. T.F. \& Paucha. E. J. virol. 62, $1814-1818(1988)$.

2. Stabel. S. Arges, P. \& Philipson, I. EMBO J. 4, 2329-2336 (1985).

Phelps, W.C., Lee. C. L., Munger, K. \& Howley, P.M. Cell 53, 539-547 (1988).

4. Deaprio J et al Cell (in the press).

Whyte, P. et al. Nature 334, 124-129 (1988)

Whyte, P. ef al. Nature 334, 124-129 (1988).

Bister, K., Trachmann. C., Jansen, H.W..
Patschinsky, T. Oncogene 1, 97-109 (1987).

Russel. P. and Nurse. P. (ell 49, 569-576 (1987)

\section{Reversal of acidification}

SIR-Battarbee et al.' recently provided a reassuring account, based on diatom and lake-water records, that lake acidification in Scotland has either been reduced or has been reversed because of reductions in $\mathrm{SO}_{2}$ emissions since 1970. Our recent modelling results simulating long-term changes in the acidity of Scottish lake and stream waters ${ }^{2 \times}$ also suggest a recent

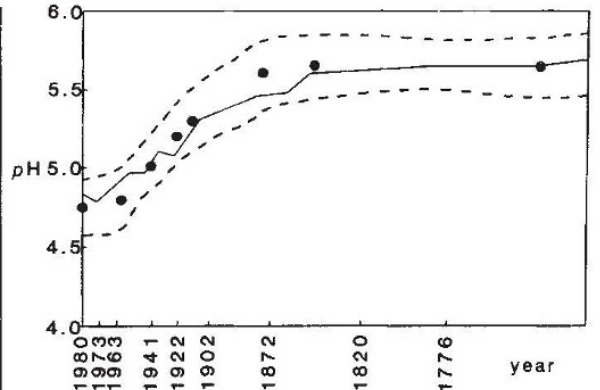

Fig. 1 Round Loch of Glenhead simulation of $p \mathrm{H}$ compared with palaeoecological data Solid line, palaeoecological reconstruction; dashed line, MAGIC reconstruction with 95\% confidence bounds; dot, MAGIC reconstruction.

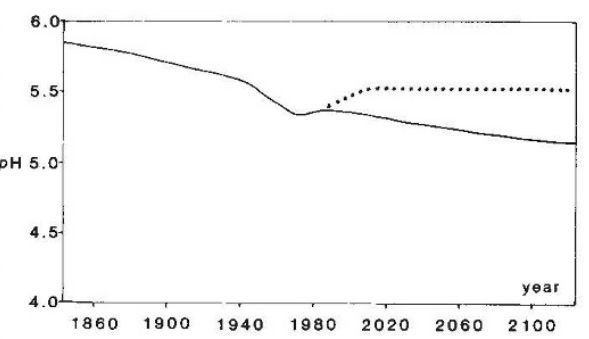

Fig. 2 Dargall Lane stream $p$ H MAGIC simulation ${ }^{7}$. Solid line, prediction with future emissions at 1985 values; dotted line, prediction with emissions declining a further $50 \%$ from 1985 values over the next 20 years.

\section{stabilization for some catchments.}

Figure 1, for example, shows a $p H$ simulation for Round Loch of Glenhead (south-west Scotland), using the MAGIC model, which compares well with the diatom reconstructed $p \mathrm{H}^{4}$. Figure 2 shows the $p \mathrm{H}$ response for Dargall Lane, a stream draining into Loch Dee in the Galloway region of south-west Scotland, which shows similar declines over the past 100 years up to the $1970 \mathrm{~s}$. The model response also indicates a recovery in the 1980 s which corresponds closely with the recovery of Battarbee et al.

Long-term predictions with the MAGIC model suggest that the recovery or stabilization is probably temporary, provided recent (1985) deposition levels are maintained. Qualitatively, this result may seem surprising. But the predicted decline results from the low rate of weathering and the highly base-depleted cation-exchange store which continues to be depleted at current acidic-oxide deposition rates. To avoid further declines in stream $p \mathrm{H}$ and ensure a significant recovery, for Dargall Lane, larger reductions in deposition of acidic oxides are required. To stabilize stream $p \mathrm{H}$ to provide a very modest improvement at Dargall Lane, for example, a further 50 per cent reduction in deposition from 1985 levels is needed, according to the estimates in Fig. 2.

Different catchments will show differ- 